\section{Original Article}

Korean J Transplant 2021;35:33-40 https://doi.org/10.4285/kjt.20.0052

\section{$\mathrm{KJT}$ \\ KOREAN JOURNAL OF TRANSPLANTATION}

\title{
Clinical significance of de novo donor-specific antibody in kidney transplant recipients with chronic antibody-mediated rejection
}

\author{
Woo Yeong Park ${ }^{1,2}$, Yaerim Kim ${ }^{1,2}$, Jin Hyuk Paek ${ }^{1,2}$, Kyubok Jin $^{1,2}$, Seungyeup Han ${ }^{1,2}$ \\ ${ }^{1}$ Department of Internal Medicine, Keimyung University School of Medicine, Daegu, Korea \\ ${ }^{2}$ Keimyung University Kidney Institute, Daegu, Korea
}

Received October 27, 2020

Revised December 14, 2020

Accepted December 15, 2020

Corresponding author: Seungyeup Han Division of Nephrology, Department of Internal Medicine, Keimyung University Dongsan Hospital, Keimyung University School of Medicine and Keimyung University Kidney Institute, 1035

Dalgubeol-daero, Dalseo-gu, Daegu 42601 , Korea

Tel: +82-53-258-7712

Fax: +82-53-258-4739

E-mail: hansy@dsmc.or.kr

(c) The Korean Society for Transplantation This is an Open Access article distributed under the terms of the Creative Commons Attribution Non-Commercial License (http://creativecommons.org/licenses/ by-nc/4.0/) which permits unrestricted non-commercial use, distribution, and reproduction in any medium, provided the original work is properly cited.
Background: Chronic antibody-mediated rejection (CABMR) is an important cause of late graft loss. De novo donor-specific antibody (dnDSA) is an important prognostic factor for long-term allograft outcomes. However, the prognosis of CABMR based on the presence of dnDSA is uncertain.

Methods: We retrospectively analyzed 35 kidney transplant recipients with CABMR between 2010 and 2018. Fourteen recipients had no detectable DSA, and 21 recipients had detectable DSA. We investigated the pathologic findings at diagnosis of CABMR, allograft function 12 months later, related factors for allograft failure, and allograft survival rate based on the presence of dnDSA.

Results: The pathologic findings showed that acute and chronic changes were more severe in the dnDSA (+) group than in the dnDSA (-) group. There was no significant difference in the allograft function 12 months after the diagnosis of CABMR and in the amount of proteinuria at diagnosis between the two groups. However, the death-censored graft survival rate was lower in the high-proteinuria group than in the low-proteinuria group in both groups. The treatment rate of recipients was higher in the dnDSA (+) group than in the dnDSA (-) group; however, there was no significant difference in the death-censored graft survival rate between the two groups.

Conclusions: Although the effect of dnDSA on the prognosis of CABMR is not clear, it would be important not to neglect treatment for CABMR with risk factors for allograft failure even without dnDSA. Continuous and rigorous surveillance of DSA and allograft function is needed in patients with CABMR.

Keywords: Kidney transplantation; Graft rejection; Antibodies; Risk factor; Treatment

\section{INTRODUCTION}

Chronic antibody-mediated rejection (CABMR) is an important cause of late graft loss. CABMR is defined by the Banff classification as follows: (1) morphologic evidence of chronic tissue injury, (2) evidence of current or recent antibody interaction with the vascular endothelium, and (3) serologic evidence of donor-specific antibodies (DSAs) to the human leukocyte antigen (HLA) or other antigens [1]. Since CABMR is known to be an extension of the acute antibody-mediated rejection that is not treated, pathologic findings are very important for the differentiation, diagnosis, and prediction of prognosis [2,3]. Also, the presence of DSA during the diagnosis of CABMR is emphasized in 


\section{HIGHLIGHTS}

-We investigated the clinical outcomes of chronic antibody-mediated rejection (CABMR) based on the presence of de novo donor-specific antibody (dnDSA).

- Pathologic findings showed that acute and chronic change was more severe in the dnDSA (+) group than in the dnDSA (-) group.

- The treatment rate of recipients was higher in the dnDSA (+) group than in the dnDSA (-) group, but, there was no difference of prognosis between the two groups.

- Continuous and rigorous surveillance of DSA and allograft function is needed in patients with CABMR.

the Banff classification [4,5]. Recently, it has been reported that de novo DSA (dnDSA) is an independent risk factor for allograft failure [6]. However, the prognosis of CABMR based on the presence of dnDSA is uncertain. Therefore, we investigated the clinical outcomes of CABMR based on the presence of dnDSA.

\section{METHODS}

\section{Human and Animal Rights}

We conducted this study in compliance with the principles of the Declaration of Helsinki. The study protocol was reviewed by the Institutional Review Board of Keimyung University Dongsan Medical Center, and they approved this study (IRB No. 2018-12-033). The Institutional Review Board waived the requirement for informed consent because the patients' data were used retrospectively for research, except for important personal information, which the individual was identified by, and was explained to all the donors' families and all recipients before kidney transplant (KT). Therefore, this study did not contain any identifiable personal information, except for the clinical process and outcome as a retrospective medical record study.

\section{Study Design}

We retrospectively analyzed 35 kidney transplant recipients (KTRs) diagnosed with CABMR between 2010 and 2018. We excluded KTRs with positive crossmatching prior to $\mathrm{KT}, \mathrm{ABO}$-incompatible $\mathrm{KT}$, and those whose data were insufficient. We divided the study population into two groups as follows: $14 \mathrm{KTRs}$ without and $21 \mathrm{KTRs}$ with dnDSA. We defined KTRs without dnDSA as KTRs with undetectable donor-specific anti-HLA antibodies and KTRs with dnDSA as KTRs with detectable donor-specific anti-HLA antibodies. We investigated the baseline characteristics of the study population, pathologic findings at the time of diagnosis of CABMR, change in allograft function before the diagnosis, and at $1,3,6$, and 12 months after the diagnosis, the amount of proteinuria at the time of diagnosis, response to treatment for CABMR, and allograft survival rate based on the presence of dnDSA.

\section{Immunosuppression Protocols}

We used basiliximab (20 mg on days 0 and 4, Simulect; Novartis, Basel, Switzerland) for KTRs with low immunologic risk and anti-thymocyte globulin (thymoglobulin; Sanofi Genzyme, Cambridge, MA, USA; $1.5 \mathrm{mg} / \mathrm{kg}$ on day 0 and 1.0 $\mathrm{mg} / \mathrm{kg}$ on days 1 through 3 ) for KTRs with high immunologic risk as immunosuppressants for induction treatment. We used cyclosporine (3 mg/kg, twice a day; Sandimmun; Novartis) or tacrolimus $(0.05 \mathrm{mg} / \mathrm{kg}$, twice a day, Prograf; Astellas Pharma Inc., Toyama, Japan), prednisolone (30 $\mathrm{mg}$, once a day), and mycophenolate mofetil (750 or 1,000 mg, twice a day, CellCept; Hoffmann-La Roche Inc., Nutley, $\mathrm{NJ}$, USA) as immunosuppressants for maintenance therapy. The treatment protocol for CABMR was as follows: high-dose intravenous immunoglobulin (IVIG; $2 \mathrm{~g} / \mathrm{kg}$ ) after a single dose of rituximab $\left(375 \mathrm{mg} / \mathrm{m}^{2}\right)$ infusion, and treatment with (2-3 sessions) or without plasmapheresis according to the pathologic findings, allograft function, and willingness to undergo the therapy.

\section{Demographic and Clinical Data}

We investigated the age of donors and recipients at the time of CABMR diagnosis, sex of the donors and recipients, KT type, frequency of $K T$, dialysis type prior to $K T$, dialysis vintage, causes of end-stage renal disease, the number of HLA mismatches, immunosuppressants for induction and maintenance treatment, previous biopsy-proven acute rejection (BPAR), the amount of proteinuria at the time of diagnosis of CABMR, panel reactive antibody (PRA) class I or II $>50 \%$, positive DSA class I or II, mean fluorescence index (MFI) value, and pathologic findings. Allograft protocol biopsies were performed at 12 months after $\mathrm{KT}$, and indication biopsies were performed at the time of allograft dysfunction or if the KTR had persistent proteinuria. All BPARs were the result of an indication biopsy in this study. Allograft biopsies were analyzed using the Banff 
2017 classification, which defined CABMR as follows: (1) morphologic evidence of chronic tissue injury, (2) evidence of current/recent antibody interaction with the vascular endothelium, and (3) serologic evidence of DSAs to the HLA or other antigens [1]. Allograft function was measured as the estimated glomerular filtration rate (eGFR) based on the modification of diet in the renal disease (MDRD) formula before the diagnosis of CABMR, at the time of diagnosis of CABMR, and at $1,3,6$, and 12 months after diagnosis or treatment. Proteinuria was measured using the spot urine protein-creatinine ratio. We examined PRA screening and identification (class I and class II) before KT and annually after $\mathrm{KT}$ to evaluate KTRs with high immunological risks. We defined KTRs with PRA $>50 \%$ as high-risk immunological patients. DSA was analyzed with a Luminex Single Antigen assay, using LABscreen Single Antigen HLA class I and class II (One Lambda, Canoga Park, CA, USA) according to the manufacturer's manual prior to KT and at 1,3 , 6 , and 12 months and annually after KT or at the time of diagnosis and monthly after the diagnosis or treatment

Table 1. Comparison of clinical and laboratory parameters based on detectable de novo DSA

\begin{tabular}{|c|c|c|c|}
\hline Variable & $\begin{array}{c}\text { Undetectable } \\
\text { de novo DSA }(n=14)\end{array}$ & $\begin{array}{c}\text { Detectable } \\
\text { de novo DSA }(n=21)\end{array}$ & P-value \\
\hline Recipient age at diagnosis (yr) & $49.0 \pm 11.7$ & $50.6 \pm 13.4$ & 0.723 \\
\hline Recipient sex (male:female) & $8(57): 6(43)$ & $16(76): 5(24)$ & 0.283 \\
\hline Donor age at diagnosis (yr) & $44.1 \pm 11.7$ & $43.5 \pm 14.6$ & 0.907 \\
\hline Donor sex (male:female) & $7(50): 7(50)$ & 10 (48):11 (52) & 1.000 \\
\hline KDPI score $(\%)$ & $53.5 \pm 23.3$ & $82.3 \pm 17.7$ & 0.208 \\
\hline Dialysis duration (mo) & $41.6 \pm 48.9$ & $35.6 \pm 44.1$ & 0.706 \\
\hline Donor type (living:deceased) & $9(64): 5(36)$ & $15(71): 6(29)$ & 0.721 \\
\hline Cause of end-stage renal disease & & & 0.141 \\
\hline Glomerulonephritis & $10(72)$ & $17(81)$ & \\
\hline Hypertension & $1(7)$ & $4(19)$ & \\
\hline Diabetes mellitus & $1(7)$ & 0 & \\
\hline Polycystic kidney disease & $2(14)$ & 0 & \\
\hline HLA mismatch number & $3.5 \pm 1.7$ & $3.7 \pm 0.9$ & 0.600 \\
\hline Preformed DSA & 0 & $4(19.0)$ & 0.133 \\
\hline PRA $>50 \%$ at diagnosis of $C A B M R$ & $3(21.4)$ & $14(66.7)$ & 0.032 \\
\hline Class I DSA (A:B) at diagnosis of CABMR & NA & $3: 3$ & \\
\hline Class II DSA (DR:DQ) at diagnosis of CABMR & NA & $11: 7$ & \\
\hline Induction & & & 0.532 \\
\hline Basiliximab & $7(50.0)$ & $13(61.9)$ & \\
\hline Antithymocyte globulin & $1(7.1)$ & $3(14.3)$ & \\
\hline None & $6(42.9)$ & $5(23.8)$ & \\
\hline \multicolumn{4}{|l|}{ Main immunosuppressant } \\
\hline \multicolumn{4}{|l|}{ Tacrolimus:cyclosporine } \\
\hline At KT & $11(78.6): 3(21.4)$ & $18(85.7): 3(14.3)$ & 0.664 \\
\hline At diagnosis & $12(85.7): 2(14.3)$ & $18(85.7): 3(14.3)$ & 1.000 \\
\hline After diagnosis or treatment & $12(85.7): 2(14.3)$ & $17(81.0): 4(19.0)$ & 0.642 \\
\hline Previous BPAR & $3(21.4)$ & $5(23.8)$ & 1.000 \\
\hline Coexistence of TCMR & $1(7.1)$ & $1(4.8)$ & 1.000 \\
\hline Time from KT to development of de novo DSA (mo) & NA & $91.6 \pm 77.4$ & \\
\hline Time from $\mathrm{KT}$ to diagnosis of CABMR (mo) & $101.6 \pm 59.6$ & $90.3 \pm 72.9$ & 0.634 \\
\hline
\end{tabular}

Values are presented as mean \pm standard deviation or number (\%).

DSA, donor-specific antibody; KDPI, kidney donor profile index; HLA, human leukocyte antigen; PRA, panel reactive antibody; CABMR, chronic antibodymediated rejection; NA, not applicable; KT, kidney transplantation; BPAR, biopsy-proven acute rejection; TCMR, T-cell mediated rejection. 
of CABMR. We defined DSA that existed before KT as preformed DSA, and when DSA occurred newly during the follow-up period after KT without preformed DSA or was different from preformed DSA was defined as dnDSA.

\section{Statistical Analysis}

Continuous variables were analyzed using the Mann-Whitney U-test, and categorical variables were analyzed by the chi-square or Fisher's exact test. Graft and patient survival rates were evaluated using the Kaplan-Meier analysis with the log-rank test. Univariate and multivariate analyses with Cox regression analysis were performed to investigate the risk factors for allograft failure. P-values $<0.05$. were considered statistically significant. Statistical analysis was performed using SPSS ver. 18 (SPSS Inc., Chicago, IL, USA).

\section{RESULTS}

\section{Baseline Characteristics of the Study Population}

The mean age of KTRs at the time of diagnosis of CABMR was $50 \pm 13$ years, and 24 patients $(68.6 \%)$ were male. Living donor KT occurred in 24 patients $(68.6 \%)$, and 30 patients $(85.7 \%)$ were the first $\mathrm{KT}$ recipients. All patients underwent hemodialysis before $\mathrm{KT}$, and 21 patients (77.1\%) had chronic glomerulonephritis as the primary renal disease. The main immunosuppressants used were tacrolimus (30, 85.7\%) and cyclosporine (5, 14.3\%). Eight patients $(22.9 \%)$ experienced BPAR prior to the diagnosis of CABMR. Median proteinuria at the time of diagnosis of CABMR was $1.1 \mathrm{~g}$ (interquartile range [IQR], 0.3-2.8 g). The time from $\mathrm{KT}$ to the development of dnDSA was $91.6 \pm 77.4$ months. The time until the diagnosis of CABMR after transplantation was 74.9 months (IQR, 42.8-142.6 months).

Table 2. Comparison of pathologic findings and clinical outcomes based on detectable de novo DSA

\begin{tabular}{|c|c|c|c|}
\hline Variable & $\begin{array}{c}\text { Undetectable } \\
\text { de novo DSA }(n=14)\end{array}$ & $\begin{array}{c}\text { Detectable } \\
\text { de novo DSA }(n=21)\end{array}$ & P-value \\
\hline Glomerulitis (g score >1) & $6(46.2)$ & $15(71.4)$ & 0.168 \\
\hline Peritubular capillaritis (ptc score >1) & $8(57.1)$ & $17(81.0)$ & 0.151 \\
\hline Microvascular inflammation ( $g+p t c$ score $>1$ ) & $11(78.6)$ & $20(95.2)$ & 0.279 \\
\hline Arteritis (v score >0) & $2(14.3)$ & $5(23.8)$ & 0.676 \\
\hline Tubulitis (t score $\geq 1$ ) & $11(78.6)$ & $16(76.2)$ & 1.000 \\
\hline Transplant glomerulopathy (cg score $\geq 1$ ) & $9(69.2)$ & $18(85.7)$ & 0.387 \\
\hline Arterial intimal fibrosis (cv score >1) & $3(21.4)$ & $7(33.3)$ & 0.704 \\
\hline IF/TA (ci+ct scores) & & & 1.000 \\
\hline $2-3$ & $6(42.9)$ & $10(47.6)$ & \\
\hline$\geq 4$ & $8(57.1)$ & $11(52.4)$ & \\
\hline Positive C4d & $6(42.9)$ & $10(47.6)$ & 1.000 \\
\hline \multicolumn{4}{|l|}{ Allograft function } \\
\hline \multicolumn{4}{|l|}{ MDRD eGFR $\left(\mathrm{mL} / \mathrm{min} / 1.73 \mathrm{~m}^{2}\right)$} \\
\hline 1 Month before diagnosis & $43.8 \pm 10.5$ & $45.7 \pm 13.5$ & 0.654 \\
\hline At diagnosis & $34.3 \pm 9.5$ & $38.8 \pm 12.3$ & 0.261 \\
\hline 1 Month after diagnosis or treatment & $33.6 \pm 10.6$ & $44.1 \pm 18.4$ & 0.043 \\
\hline 3 Months after diagnosis or treatment & $31.3 \pm 11.2$ & $44.9 \pm 18.3$ & 0.092 \\
\hline 6 Months after diagnosis or treatment & $29.3 \pm 10.6$ & $39.9 \pm 16.4$ & 0.033 \\
\hline 12 Months after diagnosis or treatment & $27.4 \pm 11.7$ & $36.9 \pm 19.8$ & 0.174 \\
\hline Proteinuria at diagnosis (g/day) & $1.7(0.4-5.4)$ & $1.1(0.3-2.2)$ & 0.207 \\
\hline Proteinuria ( $\geq 1.5 \mathrm{~g} /$ day) at diagnosis & $7(50.0)$ & $6(28.6)$ & 0.288 \\
\hline Rituximab+IVIG & $3(21.4)$ & $10(47.6)$ & 0.162 \\
\hline
\end{tabular}

Values are presented as number (\%), mean \pm standard deviation, or median (range).

DSA, donor-specific antibody; IF/TA, interstitial fibrosis/tubular atrophy; MDRD, modification of diet in the renal disease; eGFR, estimated glomerular filtration rate; IVIG, intravenous immunoglobulin. 


\section{Comparison of the Clinical and Laboratory Parameters Based on the Presence of Detectable dnDSA}

The mean follow-up duration was $129.0 \pm 72.7$ months. There were no significant differences in the mean age of donors and recipients, the proportion of sex distribution, KT type, Kidney Donor Profile Index score, KT number, duration of dialysis, dialysis type before KT, causes of end-stage renal disease, the number of HLA mismatches, induction and maintenance immunosuppressants, the rate of PRA $>50 \%$, and preformed DSA positivity, and the rate of previous BPAR between the dnDSA (-) and dnDSA $(+)$ groups. There was one case $(7.1 \%)$ of T-cell mediated rejection (TCMR) in the undetectable dnDSA group and one case $(4.8 \%)$ of TCMR in the detectable dnDSA group. There were no significant differences in the coexistence of TCMR between the two groups. The time from KT until the diagnosis of CABMR showed no significant difference between the two groups (Table 1).

\section{Comparison of the Pathologic Findings of Allograft, Allograft Function, Proteinuria, and Treatment Options Based on Detectable dnDSA}

Pathologic findings showed that acute changes such as glomerulitis, peritubular capillaritis, microvascular inflammation, arteritis, and tubulitis were more severe in the dnDSA (+) group than in the dnDSA (-) group; however, there was no significant difference between the two groups. Chronic changes such as transplant glomerulopathy, arterial intimal fibrosis, and interstitial fibrosis/tubular atrophy were also not significantly different between the two groups. The proportion of C4d positivity was not significantly different between the two groups (Table 2).

There was no significant difference in the allograft function at 12 months after the diagnosis of CABMR and the difference in the amount of proteinuria at diagnosis between the dnDSA (-) and dnDSA (+) groups. The use of rituximab and IVIG treatment was higher in the dnDSA (+) group than in the dnDSA (-) group (Table 2 ).

\section{Comparison of Death-Censored Allograft Survival and Risk Factors for Graft Failure Based on Detectable dnDSA} Nine patients (25.7\%) developed graft failure, including 5 $(35.7 \%)$ patients in the dnDSA (-) group and $4(19.0 \%)$ in the dnDSA (+) group. The causes of graft failure were as follows: chronic rejection, $4(28.6 \%)$ and $3(7.1 \%)$; infection, $1(7.1 \%)$ and $1(4.8 \%)$ in the dnDSA $(-)$ and dnDSA (+) groups, respectively. In the Kaplan-Meier analysis, there was no significant difference in the death-censored overall graft survival rate (Fig. 1A) and death-censored graft survival rate after the diagnosis of CABMR (Fig. 1B) between the dnDSA (-) and dnDSA (+) groups. In the subgroup analysis, the death-censored graft survival rate was lower in the high-proteinuria group than in the low-proteinuria group in both the dnDSA (-) and dnDSA (+) groups (Fig. 2 ). However, there was no significant difference in the death-censored graft survival rate between the two groups, regardless of the treatment administered (Fig. 3). On multivariate Cox regression analysis, deceased donor kidney transplantation and eGFR at 12 months after the diagnosis of CABMR were the risk factors associated with graft fail-
A

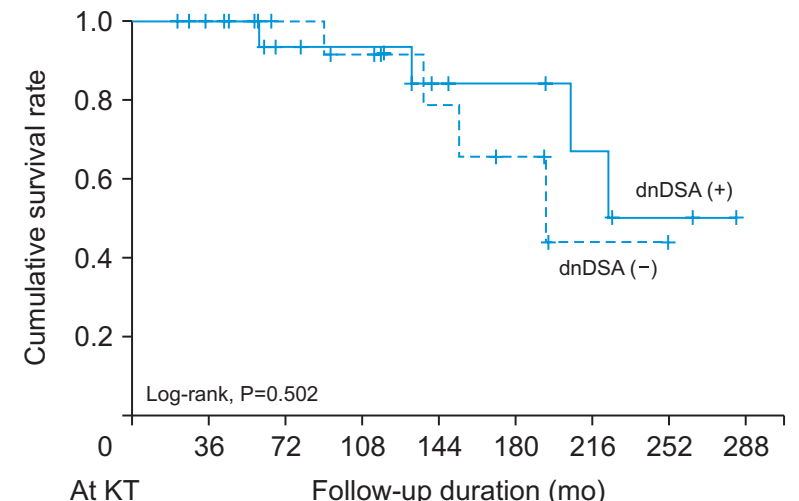

\begin{tabular}{llllllllll}
\hline $\operatorname{dnDSA}(-)$ & 14 & 14 & 14 & 13 & 12 & 11 & 10 & 10 & 10 \\
\hline $\operatorname{dnDSA}(+)$ & 21 & 21 & 20 & 20 & 19 & 19 & 18 & 17 & 17 \\
\hline
\end{tabular}

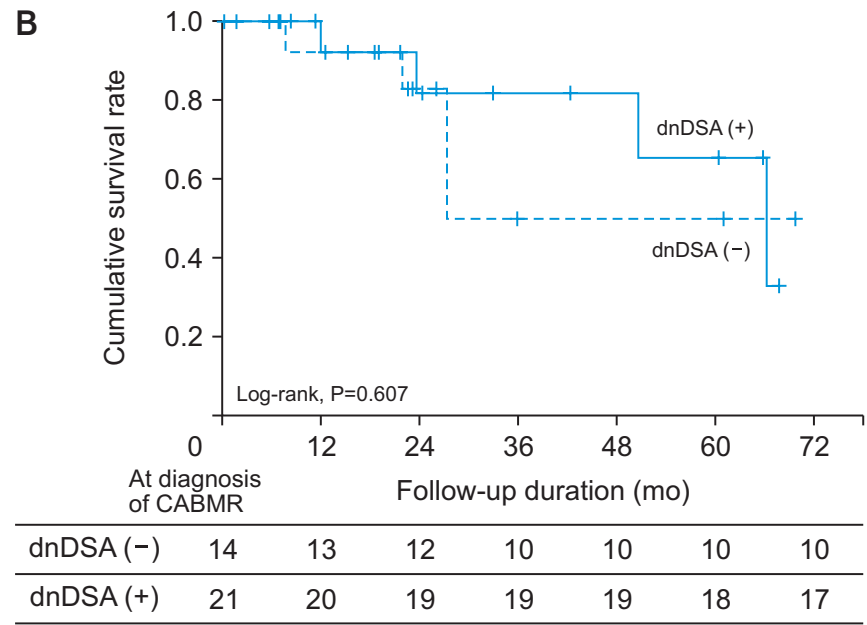

Fig. 1. Comparison of death-censored overall graft survival rate $(A)$ and death-censored graft survival rate after diagnosis of chronic antibody-mediated rejection (CABMR) (B) according to the presence of de novo donor-specific antibody (dnDSA). 
A

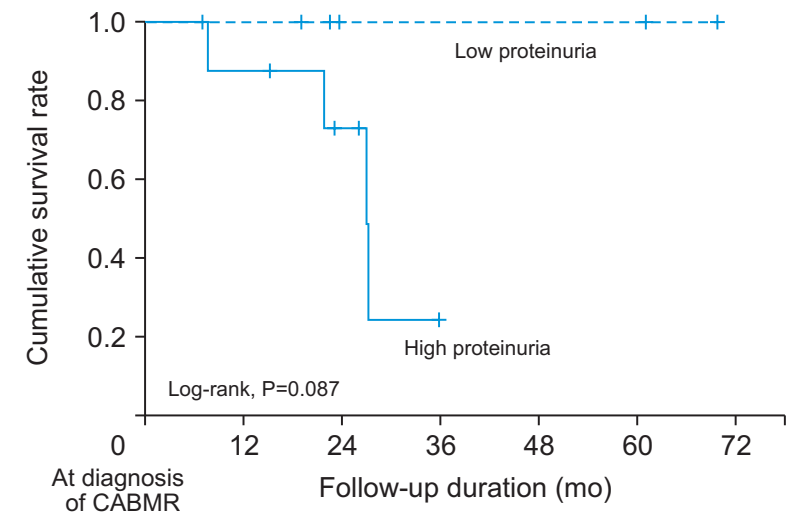

\begin{tabular}{|c|c|c|c|c|c|c|c|}
\hline $\begin{array}{c}\text { Low } \\
\text { proteinuria }\end{array}$ & 6 & 6 & 6 & 6 & 6 & 6 & 6 \\
\hline $\begin{array}{c}\text { High } \\
\text { proteinuria }\end{array}$ & 8 & 7 & 6 & 4 & & & \\
\hline
\end{tabular}

B

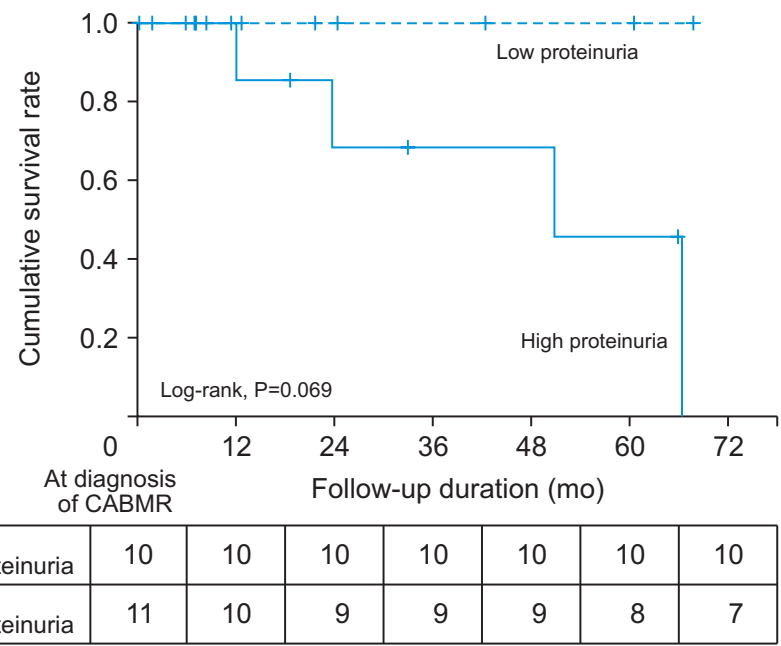

Fig. 2. Comparison of death-censored graft survival rate between the high proteinuria and low proteinuria in the $\operatorname{dnDSA}(-)$ group $(\mathrm{A})$ and $\operatorname{dnDSA}(+)$ group (B). CABMR, chronic active antibody-mediated rejection; dnDSA, de novo donor-specific antibody.

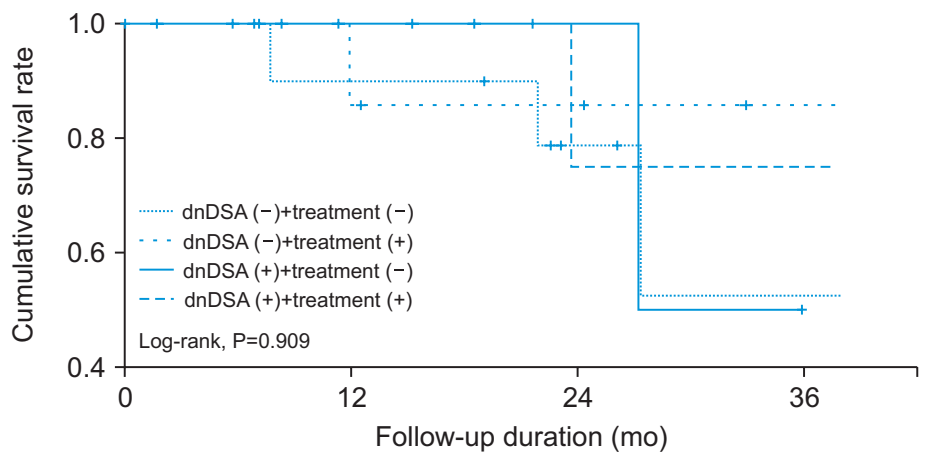

\begin{tabular}{|l|r|r|r|r|}
\hline $\operatorname{dnDSA}(-)+$ treatment $(-)$ & 11 & 10 & 9 & 8 \\
\hline $\operatorname{dnDSA}(-)+$ treatment $(+)$ & 11 & 10 & 10 & 10 \\
\hline $\operatorname{dnDSA}(+)+$ treatment $(-)$ & 3 & 3 & 3 & 2 \\
\hline $\operatorname{dnDSA}(+)+$ treatment $(+)$ & 10 & 10 & 9 & 9 \\
\hline
\end{tabular}

Fig. 3. Comparison of death-censored graft survival rate among DSA (-)+treatment $(-)$, DSA $(-)+$ treatment $(+)$, DSA $(+)+$ treatment $(-)$, and DSA $(+)+$ treatment $(+)$. dnDSA, de novo donor-specific antibody.

ure in KT after adjusting for the significant variables in the univariate analysis (hazard ratio, 57.013; 95\% confidence interval, 1.698-1,914.123, $P=0.024$; hazard ratio, 0.850; $95 \%$ confidence interval, $0.738-0.980, P=0.025$ ) (Table 3 ).

Five patients $(14.3 \%)$ died, including one $(7.1 \%)$ patient in the dnDSA (-) group and $4(19.0 \%)$ in the dnDSA (+) group. The causes of death were as follows: infection, 1 (7.1\%) and 3 (14.3\%); alveolar hemorrhage, 0 and $1(4.8 \%)$ in the dnDSA (-) and dnDSA (+) groups, respectively. In the Kaplan-Meier analysis, patient survival rates showed no significant differences between the dnDSA (-) and dnDSA $(+)$ groups (Table 3 ).

\section{DISCUSSION}

Our study found that the allograft outcome in the dnDSA $(+)$ group was similar to that of the dnDSA (-) group. Both groups showed low graft and patient survival rates. Furthermore, the pathologic findings did not differ between the two groups. In particular, the Banff 2013 classification expressed negative DSA CABMR as suspicious CABMR [7]; however, in the Banff 2017 classification, the presence of DSA was imposed, and when there was no DSA, C4d was also supported for the diagnosis of CABMR [4]. However, our study showed that there was no significant difference in the allograft outcome between the C4d (+) and C4d (-) 
Table 3. Risk factors associated with graft failure in kidney transplant recipients with CABMR

\begin{tabular}{|c|c|c|c|c|c|c|}
\hline \multirow{2}{*}{ Variable } & \multicolumn{3}{|c|}{ Univariate } & \multicolumn{3}{|c|}{ Multivariate } \\
\hline & $\mathrm{HR}$ & $95 \% \mathrm{Cl}$ & P-value & HR & $95 \% \mathrm{Cl}$ & P-value \\
\hline Recipient age & 0.999 & $0.935-1.067$ & 0.971 & & & \\
\hline Recipient male sex & 0.723 & $0.169-3.096$ & 0.662 & & & \\
\hline Donor age & 1.009 & $0.950-1.070$ & 0.777 & & & \\
\hline Donor male sex & 1.281 & $0.339-4.849$ & 0.715 & & & \\
\hline Deceased donor kidney transplantation & 6.542 & $1.246-34.359$ & 0.026 & 57.013 & $1.698-1,914.123$ & 0.024 \\
\hline Thymoglobulin induction & 1.656 & $0.175-15.707$ & 0.660 & & & \\
\hline Previous acute rejection & 0.427 & $0.053-3.450$ & 0.425 & & & \\
\hline HLA mismatches & 0.640 & $0.385-1.065$ & 0.086 & & & \\
\hline PRA $>50 \%$ at diagnosis of CABMR & 0.791 & $0.186-3.359$ & 0.751 & & & \\
\hline DSA positivity at diagnosis of CABMR & 0.511 & $0.136-1.925$ & 0.321 & 8.893 & $0.492-160.725$ & 0.139 \\
\hline eGFR at 12 months after diagnosis of CABMR & 0.893 & $0.800-0.995$ & 0.043 & 0.850 & $0.738-0.980$ & 0.025 \\
\hline Proteinuria $>1.5 \mathrm{~g} /$ day & 13.912 & $1.732-111.711$ & 0.013 & 3.355 & $0.132-85.048$ & 0.463 \\
\hline Rituximab and IVIG & 1.730 & $0.430-6.969$ & 0.441 & 0.259 & $0.022-2.984$ & 0.279 \\
\hline
\end{tabular}

CABMR, chronic antibody-mediated rejection; HR, hazard ratio; $\mathrm{Cl}$, confidence interval; HLA, human leukocyte antigen; PRA, panel reactive antibody; DSA, donor-specific antibody; eGFR, estimated glomerular filtration rate; IVIG, intravenous immunoglobulin.

subgroups in the dnDSA (-) or dnDSA (+) groups. In the subgroup analysis, we compared the clinicopathologic parameters according to graft failure, and there were no significant differences regardless of the presence of dnDSA (data not shown).

The most common treatment options for CABMR patients are rituximab and IVIG, but their effectiveness is still controversial [8-10]. Some studies have shown that bortezomib is effective for antibody-mediated rejection [11]. For the treatment of CABMR, we used the same protocol, irrespective of the presence of dnDSA or C4d, unlike in previous studies, which treated the dnDSA (-) group less aggressively than the dnDSA (+) group [12]. Furthermore, the treatment rate of recipients was higher in the dnDSA $(+)$ group than in the dnDSA (-) group. However, there was no significant difference in the death-censored graft survival rate between the two groups, regardless of the treatment. Although it has been reported that the combination treatment of rituximab and IVIG might be effective for CABMR, the results of our study showed that this is questionable regarding the presence of dnDSA. We also investigated changes in dnDSA after treatment. In our research, $21 \mathrm{KTRs}$ had dnDSA for the diagnosis of CABMR. Among them, 10 KTRs received treatment with CABMR. The MFI values of six KTRs were reduced after treatment, but dnDSA did not result in negative conversion during the follow-up period. Six KTRs had more than 5,000 MFI values or DQ DSA. However, this was not statistically significant because of the low sample size. Larger scale research and long-term follow-up periods are needed to evaluate this outcome.

It is well known that proteinuria is an important prognostic factor in allograft kidney as well as native kidney disease. Ban et al. [8] reported that proteinuria affects the prognosis of CABMR, which is consistent with the findings of our study. Moreover, in our research, the prognosis of the allograft kidney was found to be related more to the amount of proteinuria than the presence of dnDSA. In other words, the death-censored graft survival rate was lower in the high-proteinuria group than in the low-proteinuria group in both the dnDSA (-) group and dnDSA (+) groups. Furthermore, although there was no significant difference in allograft function within 12 months after the diagnosis of CABMR between the dnDSA (-) and dnDSA (+) groups, the eGFR at 12 months after the diagnosis of CABMR was the risk factor associated with graft failure, regardless of the presence or absence of dnDSA. Therefore, we should aggressively control the allograft function after the development of CABMR.

There are some limitations to our study. First, our study was retrospective, and the sample size was too small to confirm the impact of dnDSA on allograft outcome. Second, follow-up allograft biopsies were not performed. Third, we did not consider and investigate non-HLA DSA in CABMR patients with dnDSA (-).

In conclusion, although the effect of dnDSA on the prog- 
nosis of CABMR is not clear, it would be important not to neglect treatment for CABMR even without dnDSA in the case of risk factors such as heavy proteinuria, low allograft function, and deceased donor KT. Therefore, continuous and rigorous surveillance of DSA and allograft function is required in $\mathrm{CABMR}$ patients with risk factors.

\section{ACKNOWLEDGMENTS}

\section{Conflict of Interest}

No potential conflict of interest relevant to this article was reported.

\section{Funding/Support}

This study was supported by the First Research Support Project of the National Research Foundation of Korea (NRF) funded by the Ministry of Science and ICT in 2018 (NRF-2017R1C1B5076739).

This study was supported by research grant from the Korean Society for Transplantation (2021-00-01002-005).

\section{ORCID}

Woo Yeong Park https://orcid.org/0000-0003-2662-2898 Yaerim Kim https://orcid.org/0000-0003-1596-1528 Jin Hyuk Paek https://orcid.org/0000-0001-8875-1260 Kyubok Jin https://orcid.org/0000-0002-7836-8863 Seungyeup Han https://orcid.org/0000-0002-7561-6534

\section{Author Contributions}

Conceptualization: SH. Data curation: YK, JHP. Formal analysis: WYP. Funding acquisition: WYP. Investigation: KJ. Methodology: YK. Project administration: WYP, KJ. Visualization: JHP. Writing-original draft: WYP. Writing-review \& editing: WYP, SH.

\section{REFERENCES}

1. Haas M, Loupy A, Lefaucheur C, Roufosse C, Glotz D, Seron D, et al. The Banff 2017 Kidney Meeting Report: revised diagnostic criteria for chronic active T cell-mediated rejection, antibody-mediated rejection, and prospects for integrative endpoints for next-generation clinical trials. Am J Transplant 2018;18:293-307.
2. Singh N, Pirsch J, Samaniego M. Antibody-mediated rejection: treatment alternatives and outcomes. Transplant Rev (Orlando) 2009;23:34-46.

3. Levey AS, Coresh J, Greene T, Marsh J, Stevens LA, Kusek JW, et al. Expressing the Modification of Diet in Renal Disease Study equation for estimating glomerular filtration rate with standardized serum creatinine values. Clin Chem 2007;53:766-72.

4. Roufosse $C$, Simmonds N, Clahsen-van Groningen $M$, Haas M, Henriksen KJ, Horsfield C, et al. A 2018 reference guide to the Banff classification of renal allograft pathology. Transplantation 2018;102:1795-814.

5. Solar-Cafaggi $D$, Marino L, Uribe-Uribe N, Morales-Buenrostro LE. Antibody-mediated rejection in the Banff classifications of 2007 and 2017: a comparison of renal graft loss prediction capability. Transpl Immunol 2018;51:40-4.

6. Wan SS, Chadban SJ, Watson N, Wyburn K. Development and outcomes of de novo donor-specific antibodies in low, moderate, and high immunological risk kidney transplant recipients. Am J Transplant 2020;20:135164.

7. Haas M. The Revised (2013) Banff Classification for Antibody-Mediated Rejection of Renal Allografts: update, difficulties, and future considerations. Am J Transplant 2016;16:1352-7.

8. Ban $\mathrm{TH}, \mathrm{Yu} \mathrm{JH}$, Chung BH, Choi BS, Park CW, Kim YS, et al. Clinical outcome of rituximab and intravenous immunoglobulin combination therapy in kidney transplant recipients with chronic active antibody-mediated rejection. Ann Transplant 2017;22:468-74.

9. Hassan R, Gheith O. Chronic antibody-mediated rejection: review of literature. Iran J Kidney Dis 2014;8:93103.

10. Moreso F, Crespo M, Ruiz JC, Torres A, Gutierrez-Dalmau A, Osuna A, et al. Treatment of chronic antibody mediated rejection with intravenous immunoglobulins and rituximab: a multicenter, prospective, randomized, double-blind clinical trial. Am J Transplant 2018;18:927-35.

11. Gang S, Han A, Min S, Ha J, Yang J. Successful treatment of early acute antibody-mediated rejection in an human leukocyte antigen-incompatible and ABO-incompatible living-donor kidney transplant patient. Korean J Transplant 2019;33:153-8.

12. Zhang R. Donor-specific antibodies in kidney transplant recipients. Clin J Am Soc Nephrol 2018;13:182-92. 